\title{
Kidney transplantation in patients with diabetes: better than nothing
}

\author{
Chang Seong Kim
}

Department of Internal Medicine, Chonnam National University Medical School, Gwangju, Korea

Received: January 19, 2018

Accepted: January 24, 2018

\section{Correspondence to}

Chang Seong Kim, M.D.

Department of Internal Medicine, Chonnam National University Medical School, 42 Jebong-ro, Dong-gu, Gwangju 61469 , Korea

Tel: +82-62-220-6254

Fax: +82-62-225-8578

E-mail: laminion@hanmail.net

\section{See Article on Page 356-366}

In 2016 , more than 15,000 patients received dialysis in Korea, and the number of newly performed dialysis procedures is growing rapidly every year. The endstage renal disease (ESRD) incidence rate has reached 311 patients per million population per year, which is nearly double the rate 10 years ago. The prevalence of diabetes in the Korean population has also increased over the past few years. In a nationwide survey, the prevalence of diabetes in adults over age 30 years was $9 \%$ to $10 \%$ from 2000 to 2010 . However, it increased to $10 \%$ to $11 \%$ from 2011 to $2014[1,2]$. It is well known that diabetic nephropathy is a major microvascular complication of diabetes resulting from complex mechanisms that are linked to metabolic and hemodynamic changes. Diabetes is the leading cause of ESRD in Korea. In fact, $50 \%$ of all patients with ESRD were diagnosed with diabetes, according to data published in the 2016 ESRD registry of the Korean Society of Nephrology (http://www.ksn.or.kr).

Most of all, survival of patients with ESRD secondary to diabetes is worse than that of patients with other causes of ESRD. The 5-year survival rates for men and women with ESRD receiving dialysis were $60.7 \%$ and $63.7 \%$, respectively. However, the 5-year survival rate decreased to $53.9 \%$ in patients with ESRD and diabetes-related complications. This rate was even lower than the survival rate for major cancers, such as breast and colon cancer [3]. Therefore, there is currently an unmet need to improve the prognosis of patients with diabetes who are receiving dialysis.

Kidney transplantation is associated with better survival and quality of life compared to treatment with dialysis alone [4]. Strict blood glucose control and management of diabetic complications in addition to living- or deceased-donor kidney transplantation is considered the best treatment option for patients with ESRD and diabetes to improve their overall survival. However, it remains controversial whether kidney transplantation can provide similar clinical outcomes for patients with and without diabetes, especially with regard to graft survival and mortality. Previous studies have shown that graft and patient survival outcomes are poor in patients with diabetes compared to patients without diabetes after kidney transplantation [5-7]. Nevertheless, it is noteworthy that 5 -year patient survival has remained stable at a rate of $70 \%$ in patients with diabetes after living- or deceased-donor kidney transplantation. In other words, the survival rate is higher in patients with diabetes who received a kidney transplantation than in those on maintenance dialysis alone. 
Baek et al. [8] assessed clinical outcomes after living-donor kidney transplantation in patients with diabetes compared to age- and sex-matched patients without diabetes. Although urinary tract infections and cardiovascular events occurred more frequently in the patients with diabetes, the incidence of acute rejection, death-censored, non-death censored graft failure, and mortality were not significantly different between the patients with and without diabetes after living-donor kidney transplantation. Moreover, the graft failure rate and the incidence of rejection in patients with diabetes were not significantly higher than the same rates in patients without pre-existing or post-transplant diabetes mellitus. Keddis et al. [7] reported that the 5 -year mortality rate in patients with diabetes after kidney transplantation declines significantly over time, thereby narrowing the mortality rate difference between patients with and without diabetes. In addition, reduced mortality over time is more affected by the use of statins and $\beta$-blockers, and the maintenance of glycemic control after transplantation than any pre-transplantation risks. Therefore, post-transplantation care, including strict glucose control, intensive life style modifications, and cardioprotective medications are important to reduce graft failure and patient mortality, as well as to prevent the onset of post-transplantation diabetes mellitus [9]. Large prospective randomized trials are needed to evaluate the effects of glycemic control, blood pressure control, and medication therapy on clinical outcomes after kidney transplantation in patients with diabetes.

Several long-term follow-up studies showed that patients who underwent simultaneous pancreas and kidney (SPK) transplantation had superior patient and graft survival benefits compared to living-donor kidney transplantation in those with type 1 diabetes $[10,11]$. Thus, the transplant options for SPK or the pancreas after kidney transplantation not only restore renal function but also alleviate the hyperglycemic state due to diabetes [12].

In summary, there is a clear, but unmet need to improve graft and patient survival rates for patients with diabetes and ESRD. In this sense, kidney transplantation could be an irreplaceable treatment option to increase survival in patients with diabetes, regardless of the type of diabetes.

\section{Conflict of interest}

No potential conflict of interest relevant to this article was reported.

\section{REFERENCES}

1. Koo BK, Moon MK. Are we in the same risk of diabetes mellitus? Gender- and age-specific epidemiology of diabetes in 2001 to 2014 in the Korean population. Diabetes Metab J 2016;40:175-181.

2. Choi YJ, Kim HC, Kim HM, Park SW, Kim J, Kim DJ. Prevalence and management of diabetes in Korean adults: Korea National Health and Nutrition Examination Surveys 1998-2005. Diabetes Care 2009;32:2016-2020.

3. Jung KW, Won YJ, Oh CM, et al. Cancer statistics in Korea: incidence, mortality, survival, and prevalence in 2014. Cancer Res Treat 2017;49:292-305.

4. Tonelli M, Wiebe N, Knoll G, et al. Systematic review: kidney transplantation compared with dialysis in clinically relevant outcomes. Am J Transplant 2011;11:2093-2109.

5. Cosio FG, Hickson LJ, Griffin MD, Stegall MD, Kudva Y. Patient survival and cardiovascular risk after kidney transplantation: the challenge of diabetes. Am J Transplant 2008;8:593-599.

6. Tokodai K, Amada N, Kikuchi H, Haga I, Takayama T, Nakamura A. Outcomes of renal transplantation after end-stage renal disease due to diabetic nephropathy: a single-center experience. Transplant Proc 2012;44:77-79.

7. Keddis MT, El Ters M, Rodrigo E, et al. Enhanced posttransplant management of patients with diabetes improves patient outcomes. Kidney Int 2014;86:610-618.

8. Baek CH, Kim H, Baek SD, et al. Outcomes of living donor kidney transplantation in diabetic patients: age and sex matched comparison with non-diabetic patients. Korean J Intern Med 2017;33:356-366.

9. Chakkera HA, Weil EJ, Pham PT, Pomeroy J, Knowler WC. Can new-onset diabetes after kidney transplant be prevented? Diabetes Care 2013;36:1406-1412.

10. Morath C, Zeier M, Dohler B, Schmidt J, Nawroth PP, Opelz G. Metabolic control improves long-term renal allograft and patient survival in type 1 diabetes. J Am Soc Nephrol 2008;19:1557-1563.

11. Becker BN, Brazy PC, Becker YT, et al. Simultaneous pancreas-kidney transplantation reduces excess mortality in type 1 diabetic patients with end-stage renal disease. Kidney Int 2000;57:2129-2135.

12. Lindahl JP, Jenssen T, Hartmann A. Long-term outcomes after organ transplantation in diabetic end-stage renal disease. Diabetes Res Clin Pract 2014;105:14-21. 